\title{
Control Speed Ratio Electro-Hydraulic System of Continuously Variable Transmission (CVT) by Robust PI Controller
}

\author{
Sameh Bdran, Ma Shuyuan, Samo Saifullah, and Jie Huang
}

\begin{abstract}
The electro-hydraulic systems are commonly used in the motion control applications. This paper represents an implementation of robust control for an electro- hydraulic system of CVT. The robust PI controlled system for speed ratio electro-hydraulic system of CVT is designed for adjusting speed ratio of CVT by using MATLAB environment. The objective of this study was to design the controller of an electro-hydraulic system using the robust PI control system which is to maintain guarantee of the speed ratio electro-hydraulic system performance in spite of model inaccuracies and alteration. The Mathematical modeling of the electro-hydraulic system of CVT is presented in terms of equations. The robustness and tracking ability of the designed controller were demonstrated through simulations. According to the results, the robust PI controlled system for speed ratio electro-hydraulic system of CVT gives the good performance, such as rise time, settling time, overshooting.
\end{abstract}

Index Terms-Electro-hydraulic, CVT, Simulation, Robust PI controller.

\section{INTRODUCTION}

In recent years, electro-hydraulic servo (EHS) system has attracted a great interest in industrial engineering as an actuator for high performance and precision positioning applications. With a compact size and design, EHS system capable to generate high forces in fast response time and produced great durability in particular for heavy engineering system [1]. Due to the electro-hydraulic systems are used in several control applications for industry systems. Therefore the controlling of the performance characteristics of electro-hydraulic system is important.

To develop an unmanned automatic excavator system the control performance of hydraulic actuators should be guaranteed. However, hydraulic actuators with single rod cylinder have inherently severe nonlinearities that significantly affect to the command following performance of end-effect. PID control system widely used in industries is not proper to compensate the nonlinearities and it is difficult to cancel the unexpected disturbance. In this work, the robust control scheme to compensate an nonlinearity of hydraulic actuator and cancel an unexpected disturbance is proposed. The proposed control system is designed based on

Manuscript received April 5, 2013; revised May 28, 2013. This work was supported by Beijing Institute of Technology.

The authors are with the Mechatronic Center, Mechanical Engineering Beijing Institute of Technology, Beijing, 100081 China (e-mail: sameh_badran2008@yahoo.com, bitmc@bit.edu.cn, sf.samo@gmail.com, bit_huangjie@bit.edu.cn). back-stepping controller with sliding model control system. To evaluate the performance of the proposed control system, step responses were compared with a conventional SMC in low pressure area. Also, the performance of disturbance cancellation was compared applying the impact at the end of hydraulic cylinder. Through the experiments it is found that the proposed control has a better performance against parameter uncertainty and unexpected disturbance [2].

The current belt type CVT cars are mostly equipped with electro-hydraulic system. Combination of engine and continuously variable transmission (CVT) can potentially reduce fuel consumption and emissions by improving engine efficiency.

The hydraulic system of CVT model and fuzzy logic controllers are established. Two valves are introduced and analyzed corresponding of application PWM in hydraulic system. The simulation result of the complete system is good [3].

In this paper, the design of robust PID-controlled system for speed ratio an electro hydraulic system in CVT of modeling is designed by using MATLAB/Simulink.

The rest of the paper is organized as follows. In Section II, the modeling of system dynamics for the electro- hydraulic system is presented and linearized. In Section III, the design of robust control of the electro hydraulic system of speed ratio for CVT is established then implemented in MATLAB/Simulink. Section IV presents analysis of parameters control used in simulations. Simulation results for the controller Simulink models are discussed in section V. The concluding remarks are given in Section VI.

\section{Modeling of HydRAUlic DynAmics System In StATE SPACE}

Hydraulic primary cylinder of CVT is operated through flow and pressure control of speed ratio control valve (SRV) which is manipulated by electrical current of solenoid valve $I_{s o l, p}$. The oil flowing in primary cylinder is supplied by a secondary hydraulic pressure $p_{s}$ that is manipulated by electrical current of solenoid valve $I_{\text {sol,s }}$. Also it's assumed constant high supply pressure. The hydraulic actuation system of CVT is shown in Fig. 1.

In Fig. 2, the hydraulic primary circuit of CVT is shown which can be considered as a single rod hydraulic cylinder system. The motion $x_{v}$ of the speed ratio valve controls the amount flow of oil to or from the primary cylinder.

To design PID robust controller the modeling of simple single rod hydraulic actuator system will be presented later 
based on Fig. 2.

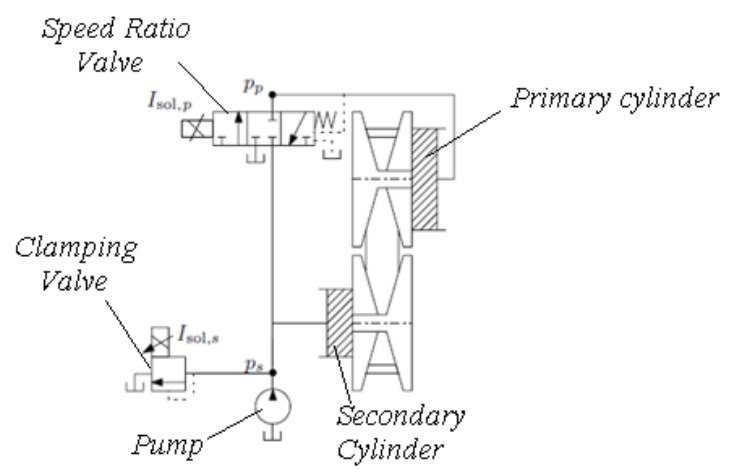

Fig. 1. Schematic of the hydraulic actuation system of CVT.

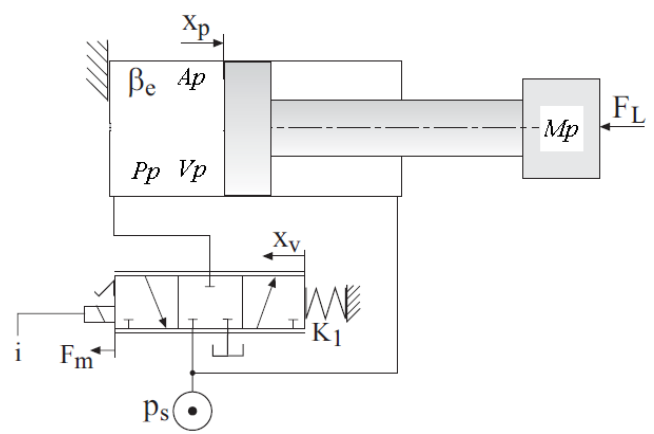

Fig. 2. The electro-hydraulic actuation system of primary pulley [4].

A mathematical model of electro-hydraulic primary pulley system is composed of the dynamics of the primary pulley system disturbed by an external load from secondary pressure $P_{s}$ via push-belt and the dynamics of a servo valve of shifting (speed ratio valve). The linearized differential equations that describe the primary pulley-valve dynamics are described as follow:

\section{A. Valve Dynamics}

According to the equation of motion of spool and the low natural frequency of valve compared to the natural frequency of primary pulley can consider the linear relation, as obtained:

$$
\Delta x_{v}(s)=K_{v} \Delta I(s)
$$

where $K_{v}$ the gain of speed ratio valve.

\section{B. Primary cylinder dynamics}

The flow through the valve can be described by:

$$
Q_{L}=Q\left(x_{v}, \Delta p\right)=C_{q} w x_{v} \sqrt{\frac{2}{\rho} \Delta p}
$$

From Fig. 2 flows $Q_{p}$ can be induced by Bernoulli's equation as follows:

$$
\begin{array}{ll}
Q_{p 1}=C_{q} w x_{v} \sqrt{\frac{2}{\rho}\left(p_{s}-p_{p}\right)} & x_{v} \geq 0 \\
Q_{p 2}=C_{q} w x_{v} \sqrt{\frac{2}{\rho}\left(p_{p}-p_{t}\right)} & x_{v}<0
\end{array}
$$

where $C_{q}$ and $w$ are flow coefficient and area gradient, $x_{v}$ is distance of spool, $P_{s}, P_{p}$ and $P_{t}$ are secondary (supply), primary cylinder and drain pressures respectively and $\rho$ is density of hydraulic fluid.

The linearized equation describing the valve flow $Q_{L}$ becomes:

$$
\Delta Q_{L}=\frac{\partial Q_{L}}{\partial x_{v}} \Delta x_{v}+\frac{\partial Q_{L}}{\partial p_{L}} \Delta p_{L}
$$

where $\frac{\partial Q_{L}}{\partial x_{v}}$ and $\frac{\partial Q_{L}}{\partial p_{L}}$ are valve flow gain and valve flow -pressure coefficient can be described by:

$$
\begin{gathered}
\frac{\partial Q_{L}}{\partial x_{v}}=K_{q}=C_{q} w \sqrt{\frac{2}{\rho}\left(p_{s}-p_{p}\right)} \\
\frac{\partial Q_{L}}{\partial p_{L}}=K_{c}=\frac{C_{q} w x_{v 0}}{2 \sqrt{\rho\left(p_{s}-p_{p 0}\right)}} \\
\Delta Q_{L}=K_{q} \Delta x_{v}+K_{c} \Delta p_{L}
\end{gathered}
$$

The pressure dynamics for primary cylinder is given by:

$$
\Delta Q_{p}=A_{p} \frac{d x_{p}}{d t}+C_{l, p} P_{p}+\frac{V_{p}}{\beta_{e}} \frac{d p_{p}}{d t}
$$

where $C_{l, p}$ and $\beta_{e}$ are internal leakage coefficient and effective bulk modulus, $A_{p}$ and $V_{p}$ are area and volume of primary cylinder distance, $P_{p}$ is the pressure inside the primary cylinder, let $\Delta Q_{p}=\Delta Q_{L}$ so:

$$
\dot{p}_{p}=\frac{\beta_{e}}{V_{p}}\left\{K_{q} x_{v}-\left(K_{c}+c_{l, p}\right) P_{p}-A_{p} \dot{x}_{p}\right\}
$$

The linearized and laplace - transformed of force equation applied on the primary pulley will be written as

$$
A_{P} \Delta P_{p}-F_{s}=M_{p} \ddot{x}_{p}+B_{P} \dot{x}_{p}+k_{p} x_{p}
$$

The $k_{p}=0$ so this lead to:

$$
\ddot{x}_{p}=\frac{1}{M_{p}}\left(-B_{P} \dot{x}_{p}+A_{P} \Delta P_{p}-F_{s}\right)
$$

The state-space model of the electro-hydraulic servo system can be shown in the following equations:

$$
\begin{aligned}
& \dot{x}=A x+B u \\
& y=C x
\end{aligned}
$$

where

$$
A=\left[\begin{array}{ccc}
1 & 0 & 0 \\
0 & -\frac{B_{p}}{M_{p}} & \frac{A_{p}}{M_{p}} \\
0 & -\frac{\beta_{e} A_{p}}{V_{p}} & -\frac{\beta_{e}\left(K_{c}+c_{l, p}\right)}{V_{p}}
\end{array}\right]
$$




$$
\begin{gathered}
B=\left[\begin{array}{cc}
0 & 0 \\
0 & -\frac{1}{M_{p}} \\
\frac{\beta_{e} K_{q}}{V_{p}} & 0
\end{array}\right] \\
C=\left[\begin{array}{ll}
1 & 0 \\
0 & 1
\end{array}\right]
\end{gathered}
$$

As seen in the above mentioned dynamic, the system is MIMO system the state variables are $x_{p}, \dot{x}_{p}$ and $P_{p}$, which has 2 outputs, $P_{p}$ - primary pulley pressure and $x_{p}-$ position of primary pulley, and 2 inputs (u), $x_{v}$ - input servo valve of the position control system, and $P_{s}$ - input servo valve of the secondary pressure control system.

\section{Robust Control Design}

The design of control systems in the presence of significant uncertainty requires the designer to seek a robust system. A system is robust when the system has acceptable changes in performance due to model changes or inaccuracies.

A system structure that incorporates potential system uncertainties is shown in Fig. 3. This model contains the sensor noise $N(s)$, the unpredicted disturbance input $T_{d}(s)$, and a process $G(s)$ with potentially unmodeled dynamics or parameters changes [5].

A control system is robust when (1) it has low sensitivities; (2) it is stable over the range of parameters variations, and (3) the performance continuous to meet the specifications in the presence of a set of changes in the system parameters.

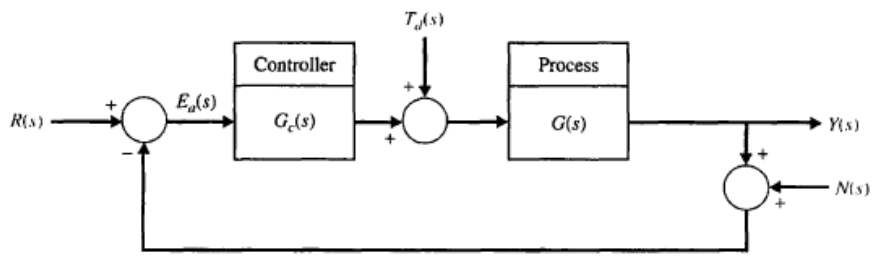

Fig. 3. Block diagram of Closed-loop control system.

The design of robust control systems is based on two tasks: determine the structure of the controller and adjusting the controller parameters to give an optimal system performance. This design process is normally done with "assumed complete knowledge" of the process. Furthermore, the process is normally described by a linear time -invariant continuous model. The structure of the controller is chosen such that system response can meet certain performance criteria [4].

The design specifications include:

- The settling time $\left(T_{s}\right)$, less than $2 \mathrm{~s}$, and

- An optimum ITAE performance for a step input.

\section{PID controller}

The PID controller has a transfer function:

$$
G_{c}(s)=K_{p}+\frac{K_{I}}{s}+K_{D} s
$$

The controller provides a proportional term $\left(K_{p}\right)$, an integration term $\left(K_{I}\right)$, and a deviation term $\left(K_{D}\right)$.

The popularity of PID controllers can be attributed partly to their good performance in a wide range of operating conditions and partly to their functional simplicity, which allows engineers to operate them in a simple, straightforward manner [4].

In order to solve the control problem using modern robust control techniques, the frequency domain characterization of the system must be known. So, The bode diagram of open loop system, closed loop without controller and the e Nyquist chart is shown in Fig. 4 and Fig. 5 respectively. From bode diagram of open loop and closed loop and the Nyquist chart its clear the system need to be controlled. Using PI controller, the ITAE design and the requirement so that:

$$
G_{c}(s)=K_{p}+\frac{K_{I}}{s}
$$

We obtain $K_{i}=2, K_{P}=5$, the examine the performance of system with varying the $K_{i}$ and $K_{p}$ will explained in the next section.

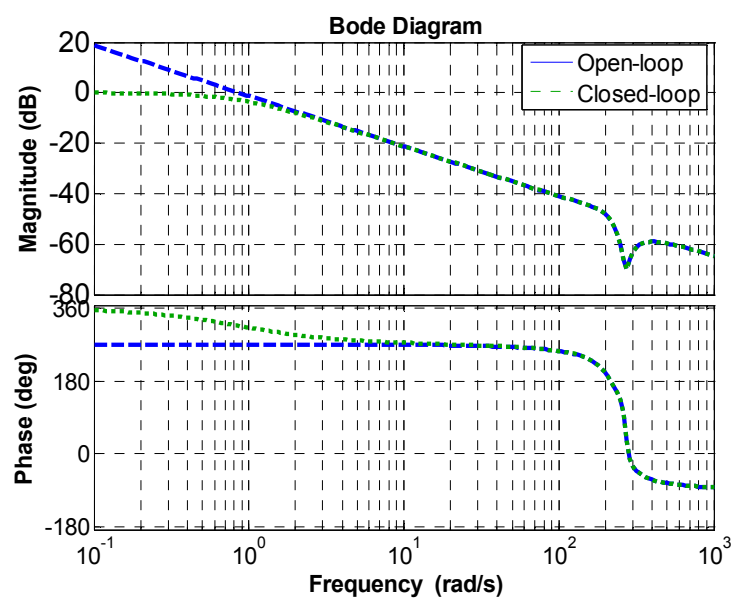

Fig. 4. The bode diagram of Open-loop (- -) and Closed-loop (...) without control system.

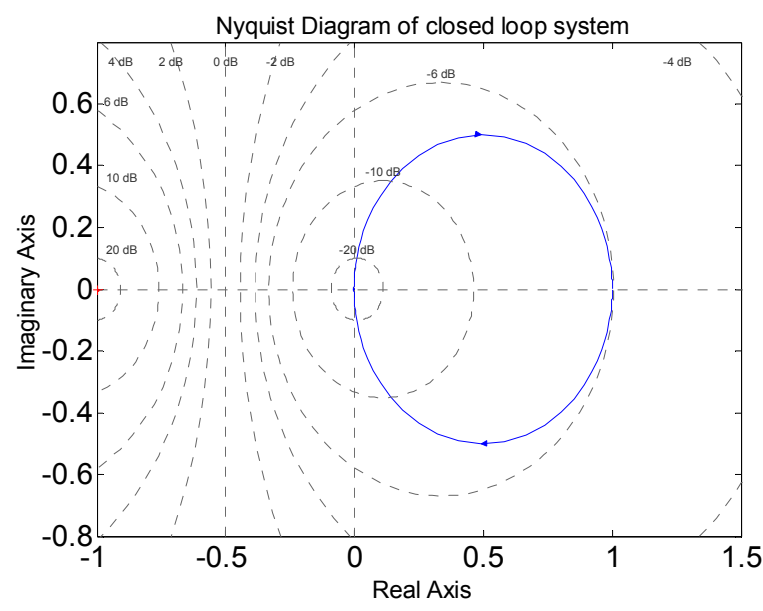

Fig. 5. The nyquist chart for the closed loop system. 


\section{Simulation Results AND Discussion}

To validate the proposed Robust PID controller scheme, the electro-hydraulic speed ratio of CVT system is simulated based on MATLAB. The parameters that required for simulation are shown in Table I.

TABLE I: THE PARAMETER OF ElECTRO HYDRAULIC SYSTEM OF CVT

\begin{tabular}{llll} 
Channels & Group 1 & Group 2 & Group $c$ \\
\hline$A_{p}$ & $301.92 \mathrm{e}^{-4}\left[\mathrm{~m}^{2}\right]$ & $\rho$ & $890\left[\mathrm{~kg} / \mathrm{m}^{3}\right]$ \\
$x_{p}$ & $-[\mathrm{m}]$ & $K_{q}$ & $0.02[\mathrm{~m} 3 / \mathrm{As}]$ \\
$V_{p}$ & $10^{-4}\left[\mathrm{~m}^{3}\right]$ & $K_{c}$ & $1.1 \times 10^{-11}$ \\
$\beta_{e}$ & $1 \mathrm{e}^{9}[\mathrm{~Pa}]$ & $m_{p}$ & $2[\mathrm{~kg}]$ \\
$C_{q}$ & $0.62[-]$ & $K_{v}$ & $0.234[-]$ \\
$w$ & $0.003[\mathrm{~m}]$ & $B_{p}$ & $1.25 \mathrm{e}^{6}[\mathrm{Ns} / \mathrm{m}]$ \\
$C_{l, p}$ & $3.4 \mathrm{e}^{-12}\left[\mathrm{~m}^{3} / \mathrm{Pa} \mathrm{s}\right]$ & & \\
\hline \hline
\end{tabular}

First, several simulations for the proposed controller system with different value $K_{P}, K_{i}$ are done and analyzed e for step amplitudes. Fig. 6 and Fig. 7 show the simulation results, it's clear the robust PI controller satisfied the requirements, the best performance its achieved at $K_{P}=5, K i=2$.

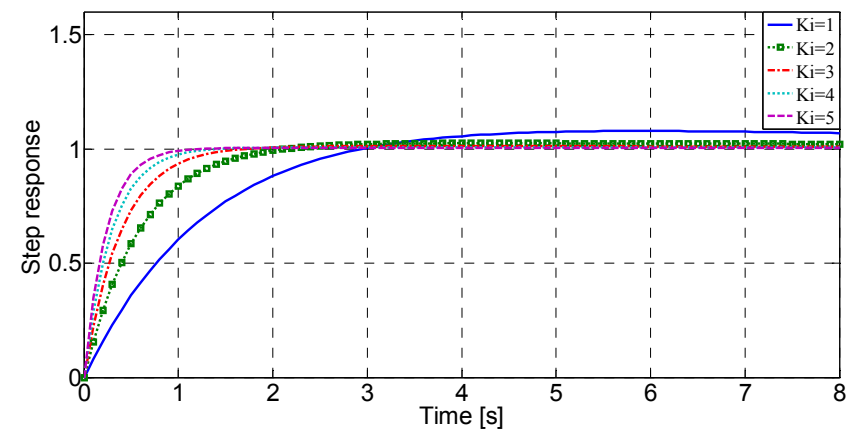

Fig. 6. The step response for different $K_{P}$ at $K_{i}=2$.

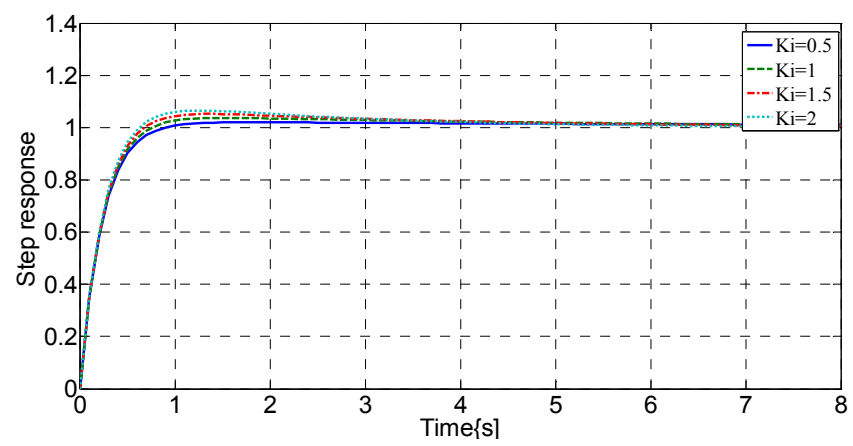

Fig. 7. The step response for different $K_{i}$ at $K_{p}=5$.

Second, the robustness of the controller can be demonstrated by applying the controller to the system in the case of erroneous parameter estimation.

The resulting bode diagrams of open loop, closed loop, closed loop with PI controller electro hydraulic system of CVT were shown in Fig. 8.

Fig. 8 also shows the proposed controller system magnitude margin, $2 \mathrm{~dB}$ and system phase margin, 300 . Obviously the proposed controlled system is robustly stable for the electro hydraulic complete CVT ratio range relative stability of the closed loop system is good. The obtained bandwidth is $1-3[\mathrm{~Hz}]$ for the speed controller.

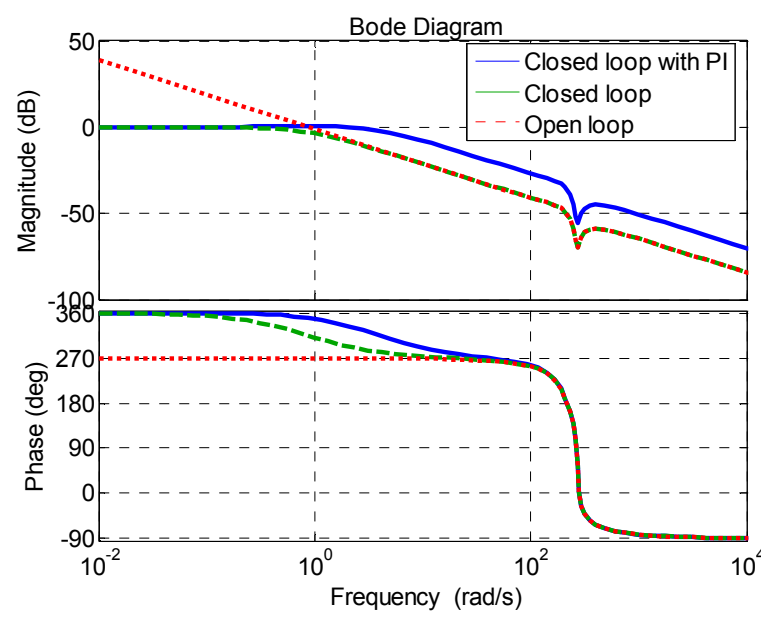

Fig. 8. The bode diagram of open loop (-), closed loop (-.-) and closed loop with PI controller (---).

Last, the resulting bode diagram of closed loop with PI controller electro hydraulic system of CVT and sensitivity is shown in Fig. 9. Also, the Bode plot of 20log T(s) and $20 \log S(s)$ is shown in Fig. 10.

From Fig. 9 and Fig. 10, we obtained the proposed control is robust and the system is low sensitive to changes.

So, the Robust PI controller for the electro hydraulic system of CVT system is give good performance and robustness.

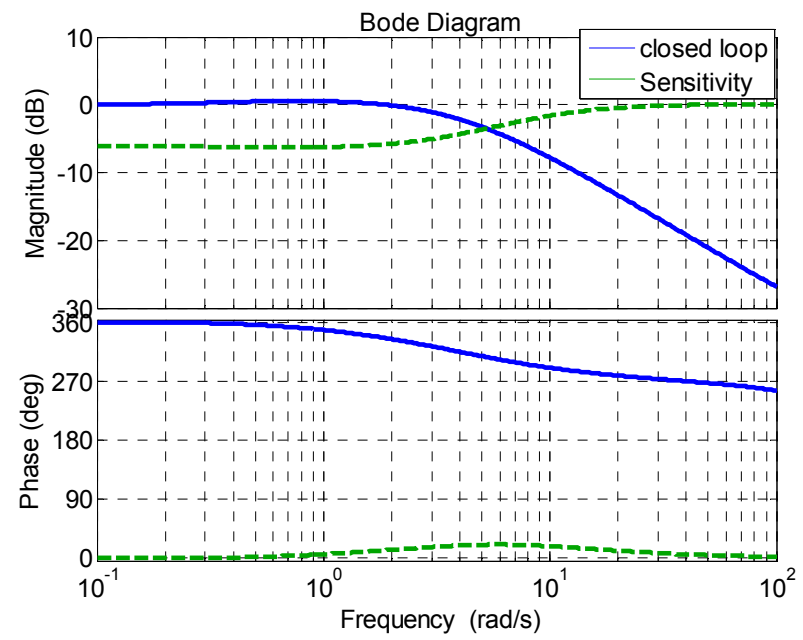

Fig. 9. The bode diagram for electro hydraulic system of CVT T(s), S(s) sensitivity.

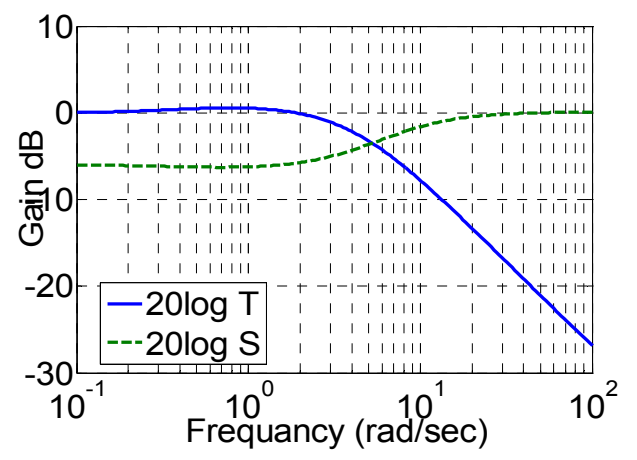

Fig. 10. The plot of closed loop system with PI T(s) and sensitivity S(s). 


\section{CONCLUSION}

The design of robust PID controller for electro-hydraulic system has been presented in this paper. The three coefficients of the PID controller were designed in order to minimize the ITAE performance index, which generate an adequate transient response to a step.

Primary pulley dynamics and electro-hydraulic models, including servo valve models were employed and described by a set of integrated system equations.

The robustness and effectiveness of the designed controllers were verified through computer simulations.

The simulation results show that the proposed robust PID controller has a good effect and robust control performance. Simulations of system with step input were presented and a good performance of the controller was obtained. Furthermore, this result is a significant enhancement of the control performance of the electro - hydraulic system.

Various robust control methods can be implemented for speed ratio electro hydraulic system of CVT such as $H_{\infty}$ synthesis, $\mu$ synthesis, etc.

\section{REFERENCES}

[1] H. E. Merrit, Hydraulic control systems, John Wiley \& Sons, Inc., New York, 1967.

[2] J. Choi, "Robust control of hydraulic actuator using back-stepping sliding mode controller," in Proc. the 28th ISARC, Seoul, Korea, pp.1261-1266, 2011.

[3] J. S. Tian and J. Su, "Hydraulic system simulation of CVT with fuzzy logic controllers," in Proc. 2004 First International Conference on Power Electronics Systems and Applications, Shanghai, pp. 136 - 141, 2004.

[4] K-E Rydberg. Hydraulic Servo Systems. [Online]. Available: www.iei.liu.se/flumes/tmhp51/filearchive/coursematerial/1.105708/H ydServoSystems_part1.pdf, 2008.

[5] R. C. Dorf and R. H. Bishop, Modern Control Systems, 11th ed., 2009.

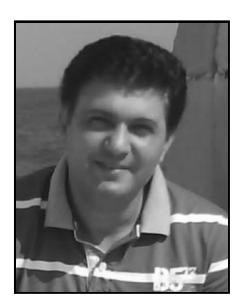

Sameh Bdran was born in Egypt, Cairo City, in 1972. $\mathrm{He}$ received the B.S. and M.S. degrees in automotive engineering from the Military Technical College, Cairo, respectively in 1996 and 2004. He is currently pursuing the Ph.D. degree in mechanical engineering at Beijing Institute of Technology, Beijing, China.

His research interest includes vehicle transmission and Mechatronics in vehicle. 